\title{
ChemComm
}

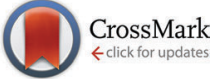

Cite this: Chem. Commun., 2015, 51, 9309

Received 4th April 2015,

Accepted 27th April 2015

DOI: $10.1039 / \mathrm{c} 5 \mathrm{cc0} 02816 \mathrm{~g}$

www.rsc.org/chemcomm

\section{Photo-triggered transformation from vesicles to branched nanotubes fabricated by a cholesterol-appended cyanostilbene $\uparrow$}

\author{
Pengyao Xing, ${ }^{\mathrm{ab}}$ Hongzhong Chen, ${ }^{a}$ Linyi $\mathrm{Bai}^{\mathrm{a}}$ and Yanli Zhao*ac
}

\begin{abstract}
Cholesterol-appended cyanostilbene was synthesized, which could self-assemble into vesicles accompanied by a aggregation-induced emission effect. Under UV light irradiation, the vesicles gradually merged together to form branched nanotubes. The self-assembly and disassembly processes could be utilized in the quantitative analysis of external stimulus, which were demonstrated by $\mathrm{H}_{2} \mathrm{O}_{2}$-selective sensing.
\end{abstract}

Vesicle trafficking, including budding and fusion, is an important and essential process in cellular conformational movement, extension and division of natural living organisms. ${ }^{1,2}$ Lipid membrane curvatures $^{3}$ allow the formation of different topological morphologies, which have been proven to be intimately connected to their functions. From a fundamental research point of view, supramolecular chemistry along with colloid and interface science has been adopted to fabricate a wide range of artificial nano-architectures in order to investigate the mechanisms of cellular differentiation and transmembrane transport. ${ }^{4}$ Membrane-based supramolecular selfassemblies, such as vesicles, tubes and lamella, that share similar bilayer membranous structures with cellular membranes, are among the most explored structures due to their applications not only in controlled release, bioimaging, sensors, templating and microreactors, but also in cellular membrane mimics. ${ }^{5}$ External stimuli could trigger morphological transformations of membranous selfassemblies accompanied with the changes of apparent properties like turbidity and luminescence. ${ }^{6,7}$ For example, vesicle to nanotube and vesicle to micelle transitions could be achieved by varying solvents, $\mathrm{pH}$ and light. ${ }^{8}$ Since vesicles normally exist as thermodynamically unstable colloids, some vesicles and polymersomes are

\footnotetext{
${ }^{a}$ Division of Chemistry and Biological Chemistry, School of Physical and Mathematical Sciences, Nanyang Technological University, 21 Nanyang Link, Singapore 637371, Singapore. E-mail: zhaoyanli@ntu.edu.sg

${ }^{b}$ School of Chemistry and Chemical Engineering and Key Laboratory of Colloid and Interface Chemistry of Ministry of Education, Shandong University, Jinan 250100, PR China

${ }^{c}$ School of Materials Science and Engineering, Nanyang Technological University, Singapore 639798, Singapore

$\dagger$ Electronic supplementary information (ESI) available: Additional synthesis and characterization data. See DOI: $10.1039 / \mathrm{c} 5 \mathrm{cc} 02816 \mathrm{~g}$
}

capable of fusing together either in a spontaneous manner or when induced by external perturbations like sonication or light irradiation. ${ }^{9}$ Vesicle to nanotube transformation via the fusion pathway offers a novel approach for building up artificial cellular membrane models to mimic membrane fusion in the vesicle trafficking. Utilizing light as the stimulus may endow the dynamic process with more properties and functions due to its clean and "green" features. During the supramolecular self-assembly process of luminescent building blocks, two distinct effects often occur, namely aggregationcaused quenching (ACQ) and aggregation-induced emission (AIE), which account for the fluorescence quenching and enhancement during the aggregation, respectively. ${ }^{10}$ AIE fluorophores are typically aromatic rings such as tetraphenylethene (TPE) and stilbene derivatives that can rotate freely in their monomeric state. Since these systems showed strong AIE in their aggregated state, they were developed as new materials for bioimaging, organic light emitting diodes (OLED), and biosensors. ${ }^{10-12}$

Herein, we have presented a cholesterol-appended cyanostilbene (CSC) that can spontaneously form vesicles in solution, and subsequently fuse together to generate branched and capped nanotubes when induced by UV light irradiation (Scheme 1). Multiple color conversions were achieved via AIE and photoisomerization-induced emission enhancement. When destroying the aggregates by external stimuli, the fluorescence originated mainly from the cis-isomer could be turned on, offering a novel route to detect the stimuli. ${ }^{13}$ In the present system, we successfully utilized this principle to detect $\mathrm{H}_{2} \mathrm{O}_{2}$ selectively under UV light irradiation.

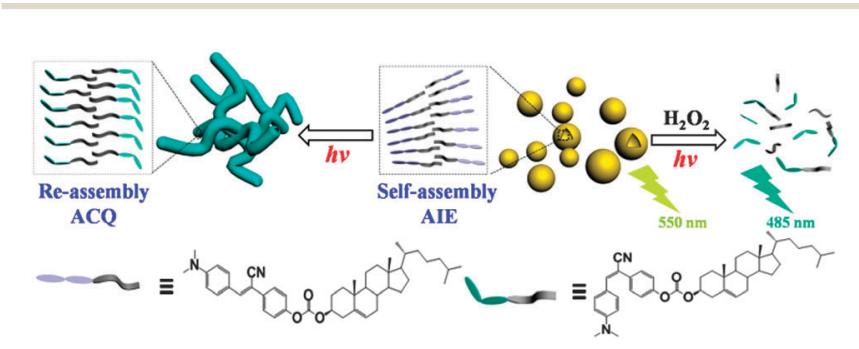

Scheme 1 Schematic representation of the controlled assembly of CSC by varying solvents as well as by UV light irradiation. 
In an organic solvent, the emission of CSC shows an overall redshift upon increasing the solvent polarity (Fig. S1, ESI $\dagger$ ), indicating that the environment can affect the aggregation of CSC. CSC has an emission peak at $\lambda_{\max }=490 \mathrm{~nm}$ in tetrahydrofuran (THF) with a blue-greenish color, while the color could hardly be observed under UV light. When adding a large fraction of water into the THF solution, a significant redshift of the emission peak $\left(\lambda_{\text {max }}=550 \mathrm{~nm}\right)$ occurred with greatly enhanced intensity (Fig. 1a). As compared to our previous report, ${ }^{11 a}$ where no obvious AIE was observed from cyanostilbene with an alkyl chain in good/poor solvents, the cholesterol fragment of CSC significantly promotes the emission. The maximum intensities of CSC with different water volume fractions $\left(f_{\mathrm{w}}\right)$ are summarized in Fig. 1c, showing two different tendencies. When $f_{\mathrm{w}}$ was below $60 \%$, the emission at $490 \mathrm{~nm}$ was further quenched. As it exceeded $60 \%$, the emission intensity started to increase, revealing a critical solvent ratio for effective molecular aggregation. A large portion of water might lead to compact aggregation, as observed by the strong emissions of samples with $90 \mathrm{vol} \%$ and $95 \mathrm{vol} \%$ water. The Commission Internationale de I'Eclairege (CIE) color coordinate was calculated as $(0.15,0.31)$ for the sample in THF, which progressively transformed into $(0.32,0.54)$ in $\mathrm{H}_{2} \mathrm{O} / \mathrm{THF}(9.5 / 0.5, \mathrm{v} / \mathrm{v})$ solution with a yellow color (Fig. S2, ESI $\dagger$ ). In comparison to the pure THF solution, the UV absorption of CSC in $\mathrm{H}_{2} \mathrm{O}$-THF mixture solution red-shifted from $380 \mathrm{~nm}$ to $390 \mathrm{~nm}$ with
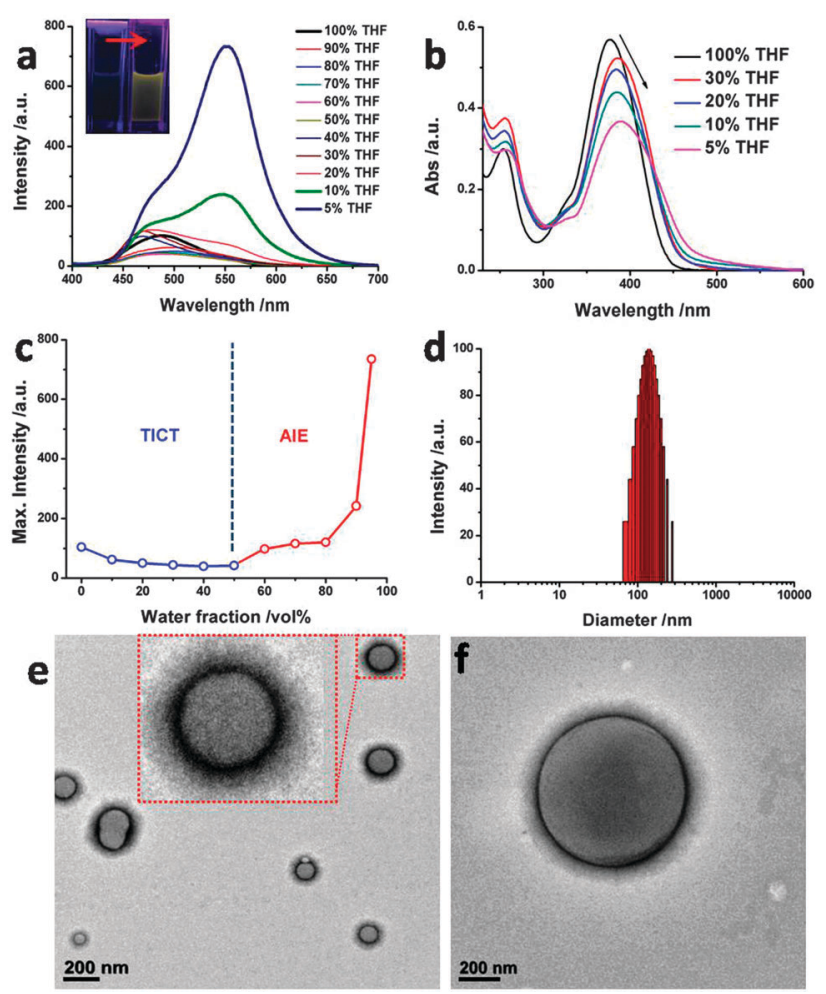

Fig. 1 (a) Emission (excitation at $380 \mathrm{~nm}$ ) and (b) absorption spectra of CSC $\left(10^{-4} \mathrm{M}\right)$ in THF with different water fractions at $298 \mathrm{~K}$. Inset of (a): macroscopic color changes upon addition of water under $365 \mathrm{~nm}$ UV light. (c) Maximum fluorescence intensity of $\operatorname{CSC}\left(10^{-4} \mathrm{M}\right)$ as a function of water fraction. (d) Hydrodynamic size distribution of CSC $\left(10^{-4} \mathrm{M}\right)$ in $\mathrm{H}_{2} \mathrm{O} / \mathrm{THF}(9 / 1, \mathrm{v} / \mathrm{v})$. TEM images of CSC in (e) $\mathrm{H}_{2} \mathrm{O} /$ dioxane $(8 / 2, \mathrm{v} / \mathrm{v})$ and (f) $\mathrm{H}_{2} \mathrm{O} / \mathrm{THF}(6 / 4, \mathrm{v} / \mathrm{v})$. The TEM samples were negatively stained by phosphotungstic acid. gradually decreasing intensity (Fig. 1b). These observations indicate that CSC forms aggregates, where the cyanostilbene moiety stacks together in a J-type $\pi-\pi$ stacking array. ${ }^{14}$ When the CSC concentration was increased, a gradual redshift of the absorption occurred (Fig. S3, $\mathrm{ESI} \dagger)$ in $\mathrm{H}_{2} \mathrm{O} / \mathrm{THF}(9 / 1, \mathrm{v} / \mathrm{v})$ solution, affording a critical aggregation concentration (CGC) of about $7 \times 10^{-5} \mathrm{M}$ (Fig. S4, ESI $\dagger$ ). It was noted that dioxane is another good organic solvent and can also be used to present similar absorption and emission changes by the addition of water (Fig. S5-S7, ESI $\dagger$ ).

To probe the diameter and morphologies of the aggregates prepared from CSC, dynamic light scattering (DLS), transmission electron microscopy (TEM), and scanning electron microscopy (SEM) were employed. The average diameter of the aggregates was determined to be $130 \mathrm{~nm}$ for the sample in $\mathrm{H}_{2} \mathrm{O} / \mathrm{THF}(9 / 1, \mathrm{v} / \mathrm{v})$ solution (Fig. 1d). Uni-lamellar vesicles with diameters of $80-200 \mathrm{~nm}$ were observed by TEM (Fig. 1e). The $f_{\mathrm{w}}$-dependent TEM and SEM studies reveal that the vesicles started to emerge when the water fraction was higher than $60 \mathrm{vol} \%$ (Fig. S8, ESI $\dagger$ ). Interestingly, when the water fraction increased, the size of vesicles gradually shrunk from initial giant vesicles (over $1 \mu \mathrm{m}$, Fig. If and Fig. S8, ESI $\dagger$ ) to small size vesicles $(50-200 \mathrm{~nm})$. It is well known that the size of the self-assemblies is determined by the curvature of molecular arrangements to some extent. ${ }^{5,15}$ Thus, the decrease of vesicle size actually reflects the increase of membrane curvature with high compactness of molecular packing. When the mixture solution has a higher water fraction, the solvophobic effect of CSC would be increased accordingly, favoring the formation of aggregates with higher curvature. The compact packing further restricts the free rotation of the cyanostilbene unit to achieve the AIE. Thermal stability of the vesicles was also evaluated (Fig. S9, ESI $\dagger$ ). Upon heating, the emission intensity of CSC shows a decreasing tendency accompanied by a slight blue shift, which may be aroused by the destruction of well-defined $\pi-\pi$ stacking arrays at high temperature.

Cyanostilbene could be switched from the initial trans $(Z$-) form to the cis (E-) form upon UV light irradiation at $254 \mathrm{~nm} .{ }^{11}$ The photoisomerization of the cyanostilbene unit in CSC was confirmed by NMR and mass spectrometry (Fig. S10 and S11, $\mathrm{ESI} \dagger$ ). After light irradiation, proton peaks of aromatic rings shifted to higher fields. Meanwhile, no new peaks could be found from the mass spectrum after UV light irradiation. According to NMR integral studies, the ratio between trans and cis isomers was 20:80, similar to other reported photoisomerizable species. ${ }^{16}$ Induced by the trans to cis isomerization of CSC in THF, the absorption peak of CSC decreased remarkably (Fig. S12, ESI $\dagger$ ), and the emission spectrum showed a blue shift to $435 \mathrm{~nm}$ with significantly increased intensity (Fig. 2a). The emitted solution color transformed from weak blue-greenish to strong blue (inset of Fig. 2a, and Fig. S14, ESI $\dagger$ ). Conformational restriction of cisisomer is responsible for the enhanced luminescence. According to our previous computational studies, ${ }^{11}$ the freely rotatable dimethylaminophenyl group with a dihedral angle of $177.9^{\circ}$ in the trans-cyanostilbene moiety enables a twisted intramolecular charge transfer (TICT) state, resulting in nonradiative decay or fluorescence quenching in low viscosity solvents like THF. Nevertheless, the free rotation is greatly restricted within the cis-isomer (Fig. 2d) with a dihedral angle of $8.9^{\circ}$. Thus, the TICT pathway 

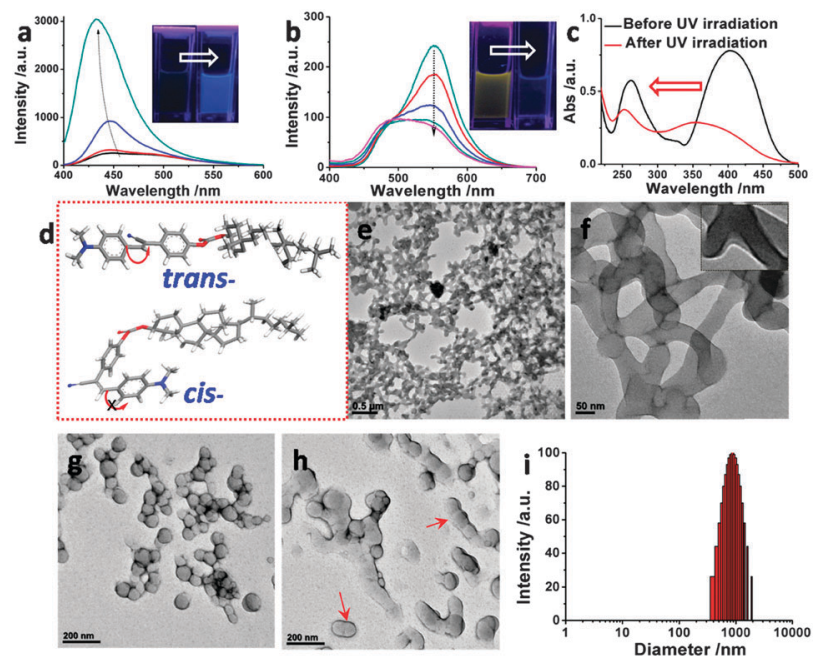

Fig. 2 Emission spectra of $\mathrm{CSC}\left(10^{-4} \mathrm{M}\right)$ in (a) pure THF and (b) $\mathrm{H}_{2} \mathrm{O} / \mathrm{THF}$ mixture $(9 / 1, v / v)$ upon UV light irradiation. Irradiation times for the curves in (b): from top to bottom 0 h, 2 h, 4 h, 6 h, 8 h. Insets of (a) and (b) stand for the macroscopic color changes under $365 \mathrm{~nm}$ UV light. (c) Absorption spectra of $\operatorname{CSC}\left(10^{-4} \mathrm{M}\right)$ in the $\mathrm{H}_{2} \mathrm{O} /$ dioxane mixture $(9 / 1, \mathrm{v} / \mathrm{v})$ before and after UV light irradiation. (d) Optimized molecular structures of trans- and cis-CSC calculated by the geometry optimization of Forcite mode in Material Studio 5.5. (e, f) Negatively stained TEM images with different magnifications for CSC $\left(10^{-4} \mathrm{M}\right)$ in the $\mathrm{H}_{2} \mathrm{O} / \mathrm{THF}$ mixture $(9 / 1, \mathrm{v} / \mathrm{v})$ after $10 \mathrm{~h}$ of UV light irradiation. $(\mathrm{g}, \mathrm{h})$ TEM images of $\mathrm{CSC}\left(10^{-4} \mathrm{M}\right)$ in the $\mathrm{H}_{2} \mathrm{O} / \mathrm{THF}$ mixture $(9 / 1, \mathrm{v} / \mathrm{v})$ after $5 \mathrm{~h}$ of UV light irradiation. (i) DLS size distribution of CSC $\left(10^{-4} \mathrm{M}\right)$ in the $\mathrm{H}_{2} \mathrm{O} / \mathrm{THF}$ mixture $(9 / 1, v / v)$ after sufficient UV light irradiation.

was suppressed in the comparatively rigid cis-isomer of CSC, giving a strong fluorescent emission.

Unexpectedly, for the self-assembled state of CSC in the $\mathrm{H}_{2} \mathrm{O} / \mathrm{THF}$ mixture (9/1, v/v) upon UV light irradiation ( 0 to $8 \mathrm{~h}$ ), the luminescence was progressively quenched, and the emitted solution color turned from yellow to blue-greenish (Fig. 2b, as well as Fig. S14 and S15, ESI $\dagger$ ). The observed abnormal quenching after light irradiation may be explained by the formation of stacked excimer species, ${ }^{10}$ resulting in ACQ. After isomerization, CSC in the self-assembled state exhibited a blue shift of the absorption band around $380 \mathrm{~nm}$ with decreased intensity (Fig. 2c, and Fig. S13, ESI $\dagger$ ), suggesting that the J-type $\pi-\pi$ stacking rearranged into an H-type one to quench the emission. TEM was employed to further examine the aggregates after photoisomerization (Fig. 2e and f). Surprisingly, entangled tubular networks instead of vesicles were observed after UV light irradiation. The enlarged TEM image showed that the networks were actually branched single wall nanotubes with slightly shorter diameters (50 to $150 \mathrm{~nm}$ ). From spherical caps of these nanotubes (Fig. S16, ESI $\dagger$ ), it was hypothesized that the nanotubes might be generated from the fusion of individual vesicles. In order to verify this assumption, the samples with insufficient UV light irradiation were investigated by TEM (Fig. $2 g$ and $h$, and Fig. S17, ESI $\dagger$ ). The merging and fusion process from vesicles to tubular morphology was observed and confirmed. Based on the TEM observations, the fusion-directed vesicle to nanotube transition could be described as follows. During UV light irradiation, the vesicles first make surface contact with each other assisted by Brownian movement, followed by the formation of figure eight or "peanut" shaped intermediates
(Fig. $2 \mathrm{~g}$ and $\mathrm{h}$ ). Further induced by the expansion of the contact area, tunnels would be constructed between linked vesicles, finally forming the nanotubes (Fig. S18, ESI $\dagger$ ). The present vesicle to nanotube transition features multiple and branched vesicle fusion, which is responsible for the formation of entangled nanotubes instead of bigger spherical vesicles. The reason is that, after light irradiation, the obtained cis-CSC favors the formation of a more planar bilayer topology with a high curvature value (e.g., nanotubes). DLS results showed broader size distribution from 200 to $2000 \mathrm{~nm}$, indicating the formation of large size aggregates of entangled nanotubes (Fig. 2i).

Basically, there are two main transition pathways, including protein assistance and perturbation-induced process, during membrane fusion in living organisms and artificial membranes of liposomes, polymersomes and vesicles. ${ }^{9,17}$ Photoisomerization and conformational change should be the major reasons for morphological transition. Firstly, isomerization changes the molecular orientation of CSC in membranes, resulting in the instability as well as deformation of vesicles. In addition, configurational conversion from the trans to cis isomer brings about a shift in solvophilic/solvophobic balance, which has a profound impact on the curvature and topologies of aggregates, as explained by the theory of molecular packing parameters. ${ }^{18}$ On top of that, the cisisomer has higher polarity and rigidity than the trans-isomer, allowing for a tighter molecular arrangement to lower the exposure of the solvophobic portion. Tighter molecular arrangement might endow it with higher curvature in favor of the contact of vesicles as well as their extension along the axis orientation, which in turn promote the tubular formation (refer to more detailed discussions in $\mathrm{ESI} \dagger$ ).

As the fluorescence of the cis-isomer was "sealed" in the nanotubes due to the ACQ effect, the destruction of the ordered structure might release the quenched fluorescence. As displayed in Fig. S19 (ESI $\dagger$ ), after adding a large amount of THF into the nanotube-containing $\mathrm{H}_{2} \mathrm{O} / \mathrm{THF}(9 / 1, \mathrm{v} / \mathrm{v})$ solution, the solution emitted stronger blue light under a UV lamp. ${ }^{11}$ The introduction of extra THF would disrupt the solvent mixture balance, thus leading to the disassociation of nanotubes to free cis-CSC with strong blue luminescence. We envisaged that the unique disassociation induced emission enhancement might provide a new route to probe the presence of external stimulus that can trigger the disassociation of nanotubes. Therefore, we utilized this character to quantitatively detect the presence of $\mathrm{H}_{2} \mathrm{O}_{2}$ assisted by UV light irradiation. UV light irradiation plays two roles in this detection: one is to photoisomerize trans-CSC to yield cis-isomer with strong emission, while the other one is to decompose $\mathrm{H}_{2} \mathrm{O}_{2}$ to generate highly energetic hydroxyl radicals $\left({ }^{\circ} \mathrm{OH}\right)$ that can destroy the self-assembled structures. As shown in Fig. 3a and Fig. S20 (ESI $\dagger$ ), UV light irradiation-treated samples exhibited enhanced fluorescence intensities around $485 \mathrm{~nm}$, corresponding to the emission of free cis-isomer in mixed solvent. Among them, the addition of $\mathrm{H}_{2} \mathrm{O}_{2}$ led to pronounced fluorescence intensity as compared with ${ }^{1} \mathrm{O}_{2}, \mathrm{ClO}^{-}$and even ${ }^{\bullet} \mathrm{OH}$ (Fenton reagent), probably due to the fact that in situ generation of • $\mathrm{OH}$ by UV light from $\mathrm{H}_{2} \mathrm{O}_{2}$ was more active than the pre-prepared Fenton reagent. After being treated with $\mathrm{H}_{2} \mathrm{O}_{2}$ along with UV light irradiation, the fluorescence intensity of the CSC solution showed a linear response to the molar equivalent of $\mathrm{H}_{2} \mathrm{O}_{2}$ (Fig. $3 \mathrm{~b}$ and c), ${ }^{19}$ suggesting that the destruction of CSC assemblies was concentration 

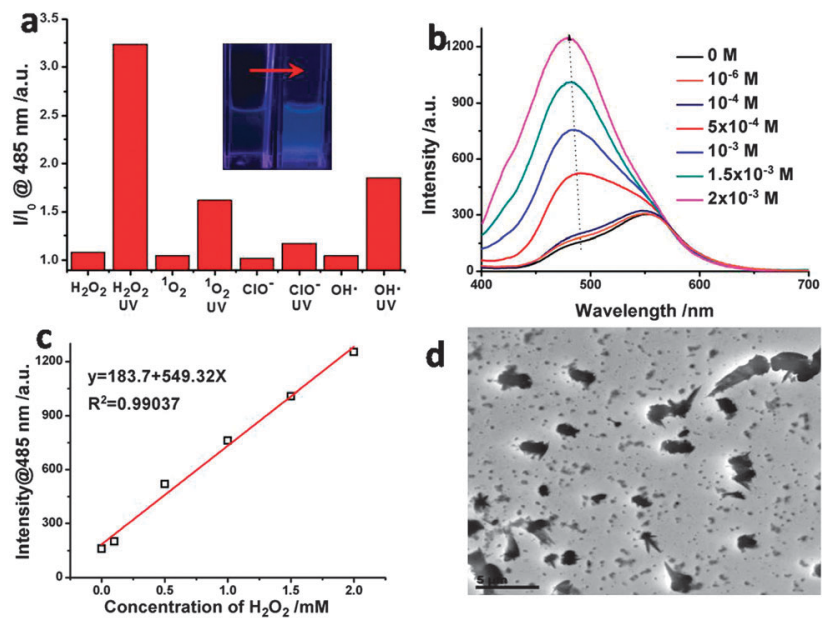

Fig. 3 (a) Ratio of fluorescence intensity (I) of CSC aggregates $\left(10^{-4} \mathrm{M}\right)$ in $\mathrm{H}_{2} \mathrm{O}$ / THF $(9 / 1, v / v)$ after different treatments (5 molar equivalents) versus original intensity $\left(I_{0}\right)$ at $485 \mathrm{~nm}$. Inset of (a) shows the solution color change under UV light. (b, c) Fluorescence intensity changes of $\mathrm{CSC}\left(10^{-4} \mathrm{M}\right)$ in $\mathrm{H}_{2} \mathrm{O} / \mathrm{THF}(9 / 1, \mathrm{v} / \mathrm{v})$ upon addition of $\mathrm{H}_{2} \mathrm{O}_{2}$ with $1 \mathrm{~h}$ of UV light irradiation. (d) TEM image of the sample after treating with $\mathrm{H}_{2} \mathrm{O}_{2}$ and UV light irradiation.

dependent with respect to $\mathrm{H}_{2} \mathrm{O}_{2}$. With the increase in irradiation time, the fluorescence intensity first increased at $485 \mathrm{~nm}$, yet it showed a decreased tendency afterwards (Fig. S21 and S22, ESI $\dagger$ ). This phenomenon indicates that, at the initial stage, the dissociation of the assemblies allows for the enhancement of fluorescence intensity due to the suppressed ACQ effect. The - OH generated from $\mathrm{H}_{2} \mathrm{O}_{2}$ would then continue to degrade CSC, reducing the amount of light emitting species in solution. Upon UV light irradiation, the absorption around $400 \mathrm{~nm}$ started to decrease, attributed to the isomerization and the degradation of CSC. After $6 \mathrm{~h}$ of irradiation, no obvious absorption peak was observed, indicating that most of CSC was degraded. When adding more $\mathrm{H}_{2} \mathrm{O}_{2}$, the absorption at $395 \mathrm{~nm}$ decreased, while a peak at $254 \mathrm{~nm}$ enhanced gradually, suggesting the breakage of more cyanostilbene moieties (Fig. S23, ESI $\dagger$ ). The TEM image (Fig. 3d) of the $\mathrm{H}_{2} \mathrm{O}_{2} / \mathrm{UV}$ treated sample displayed irregular structures, further supporting the assumptions based on the above fluorescence and absorption studies.

In summary, vesicles have been prepared from the selfassembly of cholesterol-appended cyanostilbene. Upon UV light irradiation, the emission of free molecules was greatly enhanced, while the fluorescence was quenched in the assembled state due to the conformational change caused by photoisomerization of the cyanostilbene unit. Notably, the photoisomerization could induce the vesicle fusion to form capped and branched nanotubes. Through the introduction of external stimulus to deform ordered tubular aggregates assisted by UV light irradiation, the fluorescence was recovered. This unique feature was utilized to detect $\mathrm{H}_{2} \mathrm{O}_{2}$ with good selectivity and linear response.

This work is supported by the National Research Foundation (NRF), Prime Minister's Office, Singapore, under its NRF Fellowship (NRF2009NRF-RF001-015), and Campus for Research Excellence and Technological Enterprise (CREATE) Programme-Singapore
Peking University Research Centre for a Sustainable Low-Carbon Future, as well as the NTU-A*Star Silicon Technologies Centre of Excellence under program No. 112351 0003. This work was also funded by the China Scholarship Council (CSC).

\section{Notes and references}

1 H. T. McMahon and J. L. Gallop, Nature, 2005, 438, 590.

2 E. Karatekin, J. Di Giovannic, C. Iborrac, J. Colemanb, B. O’Shaughnessye, M. Seagar and J. E. Rothman, Proc. Natl. Acad. Sci. U. S. A., 2010, 107, 3517.

3 K. Bacia, P. Schwille and T. Kurzchalia, Proc. Natl. Acad. Sci. U. S. A., 2005, 102, 3272.

4 (a) C. J. Bruns and J. F. Stoddart, Acc. Chem. Res., 2014, 47, 2186; (b) I. Budin and N. K. Devaraj, J. Am. Chem. Soc., 2012, 134, 751.

5 A. Sorrenti, O. Illa and R. M. Ortuno, Chem. Soc. Rev., 2013, 42, 8200.

6 (a) X. Ma and H. Tian, Acc. Chem. Res., 2014, 47, 1971; (b) Q. Duan, Y. Cao, Y. Li, X. Hu, T. Xiao, C. Lin, Y. Pan and L. Wang, J. Am. Chem. Soc., 2013, 135, 10542; (c) D. Guo, K. Wang, Y. Wang and Y. Liu, J. Am. Chem. Soc., 2012, 134, 10244; (d) Q. Zhao, Y. Wang, Y. Yan and J. Huang, ACS Nano, 2014, 8, 11341.

7 (a) L. A. Fielding, J. A. Lane, M. J. Derry, O. O. Mykhaylyk and S. P. Armes, J. Am. Chem. Soc., 2014, 136, 5790; (b) M. Nakahata, Y. Takashima and A. Harada, Angew. Chem., Int. Ed., 2014, 53, 3617.

8 (a) F. Versluis, I. Tomatsu, S. Kehr, C. Fregonese, A. W. J. W. Tepper, M. C. A. Stuart, B. J. Ravoo, R. I. Koning and A. Kros, J. Am. Chem. Soc., 2009, 131, 13186; (b) Y. Wang, P. Han, H. Xu, Z. Wang, X. Zhang and A. V. Kabanov, Langmuir, 2010, 26, 709; (c) X. Yan, Q. He, K. Wang, L. Duan, Y. Cui and J. Li, Angew. Chem., Int. Ed., 2007, 119, 2483; (d) T. S. Davies, A. M. Ketner and S. R. Raghavan, J. Am. Chem. Soc., 2006, 128, 6669.

9 (a) R. Lipowsky, Faraday Discuss., 2013, 161, 305; (b) Y. Zhou and D. Yan, J. Am. Chem. Soc., 2005, 127, 10468; (c) W. Chen, L. Zhai, G. Li, B. Li and J. Xu, J. Colloid Interface Sci., 2004, 278, 447; (d) W. Su, Y. Luo, Q. Yan, S. Wu, K. Han, Q. Zhang, Y. Gu and Y. Li, Macromol. Rapid Commun., 2007, 28, 1251; (e) J. Li, H. Jiang, W. Hu, H. Xia, G. Zou and Q. Zhang, Macromol. Rapid Commun., 2013, 34, 274.

10 (a) Y. Hong, J. W. Y. Lamab and B. Z. Tang, Chem. Soc. Rev., 2011, 40, 5361; (b) J. Li, Y. Li, C. Y. K. Chan, R. T. K. Kwok, H. Li, P. Zrazhevskiy, X. Gao, J. Z. Sun, A. Qin and B. Z. Tang, Angew. Chem., Int. Ed., 2014, 126, 13736.

11 (a) L. Zhu, X. Li, Q. Zhang, X. Ma, M. Li, H. Zhang, Z. Luo, H. Ågren and Y. Zhao, J. Am. Chem. Soc., 2013, 135, 5175; (b) L. Zhu, C. Y. Ang, X. Li, K. T. Nguyen, S. Y. Tan, H. Ågren and Y. Zhao, Adv. Mater., 2012, 24, 4020; (c) L. Zhu and Y. Zhao, J. Mater. Chem. C, 2013, $1,1059$.

12 (a) L. Ferrer-Tasies, E. Moreno-Calvo, M. Cano-Sarabia, M. AguilellaArzo, A. Angelova, S. Lesieur, S. Ricart, J. Faraudo, N. Ventosa and J. Veciana, Langmuir, 2013, 29, 6519; (b) J. T. vanHerpt, J. Areephong, M. C. A. Stuart, W. R. Browne and B. L. Feringa, Chem. - Eur. J., 2014, 20, 1737.

13 (a) B. C. Dickinson and C. J. Chang, Nat. Chem. Biol., 2011, 7, 504; (b) J. C. Sanchez and W. C. Trogler, J. Mater. Chem., 2008, 18, 5134.

14 S. Basak, N. Nandi, A. Baral and A. Banerjee, Chem. Commun., 2015, 51, 780.

15 L. Jiang, Y. Peng, Y. Yan and J. Huang, Soft Matter, 2011, 7, 1726.

16 (a) L. Zhu, H. Yan, K. T. Nguyen, H. Tian and Y. Zhao, Chem. Commun., 2012, 48, 4290; (b) Y. Lin, X. Cheng, Y. Qiao, C. Yu, Z. Li, Y. Yan and J. Huang, Soft Matter, 2010, 6, 902.

17 (a) V. H. S. Tellini, A. Jover, F. Meijide, J. V. Tato, L. Galantini and N. V. Pavel, Adv. Mater., 2007, 19, 1752; (b) Q. Liang, G. Chen, B. Guan and M. Jiang, J. Mater. Chem., 2011, 21, 13262; (c) B. Guan, M. Jiang, X. Yang, Q. Liang and Y. Chen, Soft Matter, 2008, 4, 1393.

18 (a) J. N. Israelachvili, D. J. Mitchell and B. W. Ninham, J. Chem. Soc., Faraday Trans., 1976, 72, 1525; (b) D. Wang, R. Dong, P. Long and J. Hao, Soft Matter, 2011, 7, 10713; (c) C. Wang, S. Yin, S. Chen, H. Xu, Z. Wang and X. Zhang, Angew. Chem., Int. Ed., 2008, 47, 9049; (d) C. Boettcher, B. Schade and J. H. Fuhrhop, Langmuir, 2001, 17, 873.

19 M. Kim, S. Ko, H. Kim, I. Shin and J. Tae, Chem. Commun., 2013, 49, 7959. 